\title{
Diretrizes brasileiras e portuguesas de proteção à criança vulnerável à violência na pandemia de COVID-19
}

\author{
Brazilian and Portuguese guidelines for protecting vulnerable children against violence in the COVID-19
} pandemic

Directrices brasileñas y portuguesas para la protección de los niños vulnerables a la violencia en la pandemia del COVID-19

Ivone Evangelista Cabral ${ }^{1,2}$ (B) Lia Leão Ciuffo ${ }^{3}$ (1) Márcia Pestana Santos ${ }^{4,6}$ (1) Yan do Rosario Nunes ${ }^{5}$ (D) Maria de Lurdes Lomba ${ }^{6}$ (I)

1. Universidade do Estado do Rio de Janeiro, Faculdade de Enfermagem, Departamento de Enfermagem Materno-Infantil. Rio de Janeiro, RJ, Brasil.

2. Universidade Federal do Rio de Janeiro, Escola de Enfermagem Anna Nery, Programa de Pós-Graduação em Enfermagem. Rio de Janeiro, RJ, Brasil.

3. Universidade Federal do Rio de Janeiro, Escola de Enfermagem Anna Nery, Departamento de Enfermagem Materno-Infantil. Rio de Janeiro, RJ, Brasil.

4. Centro Hospitalar e Universitário de Coimbra, Departamento Pediátrico. Coimbra, Portugal.

5. Universidade Federal do Rio de Janeiro, Escola de Enfermagem Anna Nery, Curso de Graduação em Enfermagem. Rio de Janeiro, RJ, Brasil.

6. Escola Superior de Enfermagem de Coimbra, Unidade de Investigação Ciências da Saúde - Enfermagem. Coimbra, Portugal.

Autor correspondente: Ivone Evangelista Cabral. E-mail: icabral444@gmail.com.

Recebido em 04/02/2021.

Aprovado em 07/05/2021.

DOI:https://doi.org/10.1590/2177-9465-EAN-2021-0045

\begin{abstract}
Resumo
Objetivo: identificar e analisar medidas de proteção à criança/adolescente vulnerável à violência na epidemia de COVID-19 no Brasil e em Portugal. Método: implementou-se a pesquisa documental de diretrizes governamentais expedidas entre março e setembro de 2020. A análise hermenêutica fundamentou-se nos preceitos da vulnerabilidade em saúde e da defesa do melhor interesse de crianças/adolescentes. Resultados: recomendaram-se ações articuladas de apoio e parcerias nacionais, locais e interinstitucionais; atuação multiprofissional, intra/intersetorial como medidas de proteção à criança/adolescente vulneráveis à violência intrafamiliar. Destacam-se o estímulo a notificação, investigação, intervenção e acompanhamento de casos. Constatam-se investimentos dos países na ampliação de canais de teleatendimento e estímulo à denúncias pela sociedade e redes sociais. Contudo, há indícios de poucos registros de casos, justificado pelas campanhas publicitárias em Portugal que incentivaram denúncias aos órgãos responsáveis. A proteção à renda e ao trabalho dos provedores da família durante o isolamento social e momento de suspensão das aulas presenciais teve a intenção de protegê-los da insegurança alimentar e do contágio da doença. Conclusão: documentos determinaram ações para instituições, profissionais de saúde, familiares e sociedade no enfrentamento da violência intrafamiliar. Na pandemia de COVID-19, é dever do Estado seguir protegendo o direito à vida e à dignidade da criança e adolescente.
\end{abstract}

Palavras-chave: Assistência Integral à Saúde; Vulnerabilidade em Saúde; Enfermagem de Atenção Primária; Serviços de Saúde da Criança; Maus-Tratos Infantis.

\begin{abstract}
Objective: To identify and analyze the protection measures for children/adolescent vulnerable to violence during the COVID-19 epidemic in Brazil and Portugal. Method: This documentary research of government guidelines issued between March and September 2020 was carried out. The hermeneutic analysis was based on the principles of health vulnerability of children/adolescents. Results: Articulated support actions and partnerships were recommended at the national, local, and interinstitutional levels. A multi-professional intersectoral action was the most recommended measure to protect those children/adolescents more vulnerable to intrafamily violence. Noteworthy are the stimulus warning, investigation, intervention, and monitoring notified cases. In addition, countries' efforts were invested in expanding the call center channels and encouraging notification violence by society members and socia networks. However, few cases were notified justified by advertising campaigns in Portugal that encouraged reporting to the responsible childhood agencies. The protection of family providers' income and work during social isolation and suspension of face-to-face classes were intended to protect them from food safety and contagious disease. Conclusion: Official documents determined actions for institutions, health professionals, family members, and society to face intrafamily violence. Regarding the effect of the COVID-19 pandemic on the family nucleus, the State must continue protecting children's and adolescents' rights to life and dignity.
\end{abstract}

Keywords: Comprehensive Health Care; Health Vulnerability; Primary Care Nursing; Child Health Services; Child Abuse.

\section{Resumen}

Objetivo: identificar y analizar medidas de protección para niños/adolescentes vulnerables a la violencia durante la epidemia de COVID-19 en Brasil y Portugal. Método: se realizó una investigación documental de las directrices gubernamentales emitidas entre marzo y septiembre de 2020. El análisis hermenéutico se basó en los principios de vulnerabilidad en salud en la niñez y la adolescencia. Resultados: se recomendaron acciones de apoyo articuladas y alianzas nacionales, locales e interinstitucionales; acción multiprofesional, intra e intersectorial como medida de protección para niños, niñas y adolescentes vulnerables a la violencia intrafamiliar. Destacan el fomento de la notificación, investigación, intervención y seguimiento de los casos. Hay esfuerzos de ambos países que invertirán en la expansión de los call center y fomentarán las quejas de los miembros de la sociedad y las redes sociales. Sin embargo, hay indicios de pocos casos denunciados, justificados por campañas publicitarias (especialmente en Portugal) que alentaron las denuncias a los órganos responsables. La protección de los ingresos y el trabajo de los proveedores familiares, durante el aislamiento social y en el momento de la suspensión de las clases presenciales tuve la intención de protegerlos del seguridad alimentar y del contagio de la enfermedad. Conclusión: documentos emitidos acciones específicas para instituciones, profesionales de la salud, familiares y sociedad en el abordaje de la violencia intrafamiliar. En la pandemia del COVID-19, es deber del Estado continuar protegiendo el derecho a la vida y la dignidad de los niños, niñas y adolescentes

Palabras clave: Atención Integral de Salud; Vulnerabilidad en Salud; Enfermería de Atención Primaria; Servicios de Salud del Niño; Maltrato a los Niños. 


\section{INTRODUÇÃO}

A pandemia de COVID-19 (Coronavirus Disease 2019), causada pelo novo coronavírus (SARS-CoV-2), resultou em mais efeitos sobre a população infantil, devido ao agravamento da crise socioeconômica mundial do que propriamente pelo impacto direto da morbidade sobre as crianças e adolescentes. As medidas de distanciamento social levaram ao fechamento de escolas, atingindo mais de 1,5 bilhão de crianças e adolescentes em todo o mundo'.

Estudo realizado pelo Fundo das Nações Unidas para a Infância (UNICEF) constatou que 104 de 136 países interromperam a oferta de serviços de proteção à criança contra a violência durante a pandemia, sendo que $83 \%$ estão localizados na América Latina ${ }^{2}$. Como esses dados se referem a países com renda baixa e média, pouco se sabe sobre a evolução da oferta desses serviços nos países da Europa centro-ocidental, onde se inclui Portugal.

Em Portugal, o fechamento das creches, infantários, escolas, atividades culturais e desportivas foi decretado (Decreto-Lei n. ${ }^{-14-G / 2020) ~ e m ~} 13$ de abril de 2020. As escolas passaram a permanecer parcialmente abertas a partir de maio de $2020^{3}$.

No Brasil, houve a suspensão total das aulas presenciais a partir de 1 de abril de 2020. Seja no regime de fechamento total ou parcial, as atividades de ensino foram transferidas da escola para o ambiente da casa, levando ao afastamento de crianças da convivência com pares e professores em caráter presencial e contínuo, uma vez que o processo de escolarização foi conduzido por videoconferência e/ou telescola. Somente no Brasil, 23 milhões de crianças e adolescentes deixaram de frequentar a escola nos ensinos básico, fundamental e médio ${ }^{3}$.

Com a transferência do ensino baseado na escola para o ambiente da casa (ou home schooling), houve um aumento do tempo de permanência e convivência da criança com a família. Enquanto não houver vacina ou tratamento seguro e efetivo, por um lado, o confinamento é uma medida protetora a saúde da criança, por outro, o prolongamento desse confinamento pode trazer repercussões negativas para este grupo populacional ${ }^{4}$.

Um dos impactos se refere à notificação de casos de violência após o fechamento das escolas no Brasil. Comparando-se dados do mês de abril de 2020 com o mesmo mês do ano anterior, constata-se uma redução de $18 \%$ nas denúncias de violência perpetradas contra crianças e adolescentes pelo Disque 100 do Disque Direitos Humanos ${ }^{5}$. Dados de denúncias de violências nos anos de 2019, comparados a 2018, evidenciam um aumento de $13,9 \%$ no número de casos. A negligência e as violências psicológica, física, patrimonial, sexual e institucional estão entre os principais tipos de violação de direitos humanos registrados. A maioria (90\%) dos indivíduos que comete algum tipo de violação é adulto na faixa etária de 18 a 59 anos. As crianças entre zero e 11 anos de idade representam $43 \%$ das vítimas ${ }^{6}$.

Em Portugal, entre as 1.492 denúncias de violência reportadas no ano de 2018, 940 eram de crianças com até 10 anos de idade, sendo que as meninas ( $n=628$ ) foram mais vitimizadas que os meninos ( $n=312$ ); e 552 eram de adolescentes entre 11 e 17 anos. Quanto à autoria da violência, a relação parental correspondeu a 27,3\%. Os locais de maior ocorrência de vitimização foram as residências $(51,2 \%)$, sendo que $20 \%$ das denúncias foram feitas pela polícia e 15\% por amigos/conhecidos/vizinhos. Esses dados revelam as condições vulnerabilizantes a que se expuseram crianças e adolescentes portugueses que, durante a pandemia de COVID 19, permaneceram em isolamento social com suas famílias em casa. A forma de denúncia mais comumente utilizada foi o contato telefônico $(75,4 \%)$, seguindo-se pelo contato presencial (30\%); as tecnologias de informação e comunicação (12\%) reforçaram os contatos por e-mail ou online (redes sociais) ${ }^{7}$. Outro dado relevante foi a institucionalização de crianças vítimas de violência. No ano de 2019, pela primeira vez em dez anos, o número de entradas de crianças e jovens no sistema foi superior ao de saídas. Algumas delas apresentaram situações de risco devido à exposição à violência doméstica, situação que aumentou quando comparado ao ano de $2018^{8}$.

A Organização Mundial da Saúde (OMS), por meio das organizações de seus continentes (OPAS nos países da América Latina), ressalta que milhões de crianças estão susceptíveis ao abuso físico, sexual, psicológico, expostas a sofrer vários tipos de lesões, incapacidades e morte. Apesar de a maioria dos países (83\%) ter dados sobre violência contra a criança, apenas $21 \%$ fizeram uso desses, com a finalidade de delineamento de estratégias concretas, visando à prevenção e à mitigação de exposição à violência. Aproximadamente $80 \%$ dos países desenvolveram planos de ação e políticas nacionais, contudo somente $1 / 5$ deles possui financiamento ${ }^{9}$.

Nesse contexto, pouco se sabe sobre quais foram as diretrizes de enfrentamento à violência contra crianças e adolescentes e que recomendações e estratégias operacionais de proteção a elas foram expedidas em documentos do Brasil e Portugal. Portanto, é relevante o desenvolvimento de estudos que busquem conhecer tais medidas adotadas por ambos os países que possuem sistemas universais de saúde e de proteção social. Ambos os países seguem a mesma doutrina de proteção à infância e adolescência, com regulamentações que asseguram o direito ao bem-estar social de crianças e adolescentes ${ }^{10}$. Em que pese as disparidades das dimensões geográficas, sociodemográficas e econômicas entre os dois países, o cumprimento desses dispositivos legais contribui para que, na prática, as iniciativas de proteção à infância e adolescência portuguesa sejam mais efetivas que as brasileiras ${ }^{11}$. Sabe-se que as crises sanitárias globais apresentam impactos geoeconômicos e políticos que afetam o estado de bem-estar social. Particularmente, nesse momento da pandemia de COVID-19, faz-se necessário se basear em dispositivos legais expedidos para assegurar a proteção de crianças e adolescentes, os quais podem ser objetos de investigação e análise. Nesse sentido, os objetivos do estudo foram identificar e analisar as medidas de proteção à criança vulnerável à violência durante a epidemia de COVID-19 no Brasil e em Portugal.

\section{MÉTODO}

Pesquisa documental, cujas fontes de dados foram dispositivos regulatórios publicados sobre medidas de proteção à criança 
vulnerável à violência na vigência da pandemia de COVID-19. $\mathrm{O}$ acesso às fontes documentais foi intencional em páginas eletrônicas de órgãos públicos de proteção à criança e adolescente tanto no Brasil quanto em Portugal. A busca dos documentos foi feita no mês de dezembro de 2020 , enquanto o recorte temporal correspondeu aos meses de março a setembro de 2020; o primeiro mês corresponde ao decreto da crise sanitária, e o último, quando se publicou a última regulamentação relacionada à proteção à criança e adolescente durante a pandemia de COVID 19.

No Brasil, buscaram-se regulamentações expedidas pelos Ministério da Mulher, da Família e dos Direitos Humanos, Ministério da Cidadania e Conselho Nacional dos Direitos da Criança e do Adolescente (CONANDA). Em Portugal, procedeu-se da mesma forma, porém nas páginas da Direção-Geral da Saúde (DGS), Comissão Nacional Promoção dos Direitos e Proteção das Crianças e Jovens (CNPDPCJ) e Sistema Nacional de Intervenção Precoce na Infância (SNIPI). Em ambos os países, na medida em que eram citados documentos e outros órgãos ou instituições nas fontes legais, rastreavam-se novas fontes para expandir o quadro documental de análise (Quadro 1).

$\mathrm{Na}$ pesquisa documental, toma-se como fonte primária de informações os documentos (leis, decreto-lei, portarias, normas técnicas, recomendações, cartilha, manuais, etc.) para responder questões e contribuir para a formulação de novas questões. Em geral, o material de análise possui potencial para atender os objetivos da pesquisa, mas não foi previamente submetido a tratamento analítico ${ }^{12}$.

A pesquisa com documentos se inicia localizando os textos que sejam pertinentes, representativos e possuam credibilidade para registrar os fatos que se deseja investigar. Os procedimentos de pesquisa envolvem três etapas: avaliação preliminar, análise documental e interpretação. Na primeira, busca-se o contexto no texto, suas dimensões, os autores, a autenticidade e confiabilidade, a natureza, as expressões-chave e a lógica do texto. No movimento da análise do documento, extraem-se significados temáticos ou significantes lexicais e buscam-se os elementos mais simples do texto.

Em seguida, procede-se a sistematização das palavras ou ideias em unidades de registro que se aproximam, codificando-as em unidades temáticas para formar temas. Os elementos significantes do conhecimento novo são apreendidos com a interpretação ou inferência, fundamentando-se em um referencial teórico de livre escolha do pesquisador.

Com base no exposto, na primeira etapa, avaliação preliminar, incluíram-se dispositivos regulatórios, manuais de procedimentos, etc., do governo de ambos os países, especificamente relacionados à proteção à criança e adolescente vulnerável à violência durante a pandemia de COVID-19 (Quadro 1), expedidos nos meses de março a setembro de 2020.

Para o estudo em foco, elegeu-se a análise hermenêutica de Paul Ricouer para o tratamento das informações extraídas do corpus documental. Nesse tipo de análise, determinam-se relações entre os núcleos de sentidos extraídos dos corpus textuais, comparando-os com diferentes situações para gerar possibilidades de interpretação hermenêutica e construir conceitos novos ${ }^{13}$.

Nesse sentido, processou-se a leitura preliminar e exaustiva dos documentos, buscando-se responder duas perguntas relacionadas à proteção de crianças e adolescentes vulneráveis às situações de violência intrafamiliar durante a pandemia de COVID-19, a saber: a) quais são as diretrizes programáticas de monitoramento e acompanhamento?; b) quais são as diretrizes programáticas de promoção e redução de dano? Dessa leitura, extraíram-se expressões-chave que contribuíram para responder questões analíticas (Quadro 2).

Para cada país, elaboraram-se quatro quadros, sendo um para a extração de conteúdos em resposta para cada pergunta, contendo três colunas (fonte documental, expressões-chave e unidades de registro (UR)). Em seguida, procedeu-se a aproximação das UR por convergência de significante lexical para formar unidades analíticas, posteriormente reagrupadas por significados comuns e singulares em núcleos de sentidos, segundo as diretrizes previstas na análise hermenêutica.

Com base nessas diretrizes, a codificação das unidades temáticas convergentes de cada país resultou em 11 quadros (seis do Brasil e cinco de Portugal), reagrupados em três quadros comuns e singulares, para ambos os países.

Na última etapa, interpretação, elegeu-se a modalidade hermenêutica para apreensão de conceitos emergentes. Para cada um dos três quadros, adotou-se a estratégia de painel de analistas (que compõem a autoria desse texto), fundamentando-se no referencial teórico da vulnerabilidade social para apreender os sentidos comuns e singulares. Ou seja, as relações sociais podem limitar a capacidade de agir das pessoas, retirando os suportes institucionais que Ihes conferem segurança social. Contudo, pode haver situações que negam a possibilidade de a pessoa exercer efetivamente seus direitos de cidadania, gerando insegurança no presente e frustação em projetos futuros ${ }^{14,15}$.

Na perspectiva dos direitos humanos, a vulnerabilidade no campo da saúde traz implícito duas dimensões coletivas (social e programática) e uma individual ${ }^{13}$. Na dimensão coletiva social na saúde de crianças e adolescentes, aplicam-se normas sociais e culturais da família, o bem-estar da família que tem acesso a emprego pleno e renda contínua e que assegure o provimento das condições materiais de existência e a defesa do melhor interesse da criança.

Ainda na dimensão coletiva, os direitos fundamentais (vida, saúde, educação, cultura, lazer e esportes, etc.) precisam ser assegurados pela família, Estado e sociedade. Incluem-se, ainda, o acesso à informação, o compromisso governamental com a saúde e o bem-estar social da família e da criança. Na dimensão coletiva programática, cabe aos governos se comprometerem com as respostas à necessidade sociais e de saúde das pessoas, formulando políticas públicas de proteção do melhor interesse da criança. Nesse sentido, precisa-se planejar e avaliar cuidados, prover de recursos (materiais e humanos) que favoreçam a implantação e sustentabilidade política; assegurar governança institucional e material, conexões e atividades intersectoriais; 
Quadro 1. Documentos orientadores da proteção de criança e adolescente expostos às situações de violência no período da pandemia de COVID-19. Brasil e Portugal, 2020.

\begin{tabular}{|l}
\hline BRASIL \\
\hline (BR.A1.CONANDA_25/03/2020). CONANDA_25/03/2020. Recomendações \\
do Conselho Nacional dos Direitos da Criança e do Adolescente - \\
Disponível em: https://crianca.mppr.mp.br/arquivos/File/legis/covid19/ \\
recomendacoes_conanda_covid19_25032020.pdf \\
(BR.A2. NT MMFDH-9/2020.) Nota Técnica (NT) n9/2020/CGDDCA/ \\
DEEVDCA/SNDCA/ Ministério da Mulher, da Família e dos Direitos \\
Humanos [MMFDH). Disponível em: https://crianca.mppr.mp.br/ \\
arquivos/File/legis/covid19/nota_tecnica_sndca_mmfdh_ref_ \\
ppcaam_03042020_covid19.pdf
\end{tabular}

BR.A3. Lei no 14.022, de 7 de julho de 2020. Disponível em: http:// www.planalto.gov.br/ccivil_03/_ato2019-2022/2020/lei/L14022.htm

BR. B4. Enfrentando a violência on-line contra adolescentes no contexto da pandemia de Covid-19. Ministério da Mulher, da Família e dos Direitos Humanos. Secretaria Nacional de Políticas para as mulheres. Disponível em:https://www.gov.br/mdh/pt-br/assuntos/ noticias/2020-2/agosto/68ENFRENTANDO_VIOLENCIA_ONLINE.pdf

(BR.B5. MMFDH. Disque 100). Disque 100. Disponível em: https:// www.gov.br/mdh/pt-br/acesso-a-informacao/disque-100-1

(BR.A6. NT_70, IPEA_22/05/2020). Nota técnica no 70, de 22 de maio de 2020. Instituto de Pesquisa Econômica aplicada (IPEA). Diretoria de Estudos e Políticas Sociais. Disponível em: https://www.ipea.gov.br/ portal/images/stories/PDFs/nota_tecnica/200522_nt_disoc_n_70.pdf BR.A7. Portaria no 86_de 1 ㅇde junho de 2020,p.12/20). Portaria no 86, de 1 o de junho de 2020. Ministério da Cidadania/Secretaria Especial do Desenvolvimento Social/Secretaria Nacional de Assistência Social. Disponível em: https://www.in.gov.br/en/web/dou/-/portaria-n-86de-1-de-junho-de-2020-259638376

BR.B8. FIOCRUZ 23/04/2020. Saúde Mental e Atenção Psicossocial na Pandemia Covid-19. Violência doméstica e familiar na COVID-19. Ministério da Saúde: Fundação Osvaldo Cruz (FIOCRUZ). FIOCRUZ. 23 Abr., 2020. Disponível em: https://portaldeboaspraticas.iff.fiocruz.br/ wp-content/uploads/2020/04/cartilha_violência_23_04.pdf

BR. B9. FIOCRUZ 08/05/2020) Saúde Mental e Atenção Psicossocial na Pandemia Covid-19. Disponível em: https://www.fiocruzbrasilia. fiocruz.br/wp-content/uploads/2020/05/crianças_pandemia.pdf BR.A8. Portaria no 59, de 22 de abril de 2020. Ministério da Cidadania/ Secretaria Especial do Desenvolvimento Social/Secretaria Nacional de Assistência Social. Disponível em: https://www.in.gov.br/en/web/ dou/-/portaria-n-59-de-22-de-abril-de-2020-253753930

\section{PORTUGAL}

PT.B1. CNPDPCJ, 2020. Comissão Nacional Promoção dos Direitos e Proteção das Crianças e Jovens (CNPDPCJ). Disponível em https:// www.dge.mec.pt/noticias/orientacoes-gerais-para-proteger-criancase-jovens-em-tempo-de-covid-19

PT.B2.SNIPI_V2_22/04/2020.SNIPI_V2_22/04/2020. Segurança Social. Disponível em http://www.seg-social.pt/documents/10152/16722120/ SNIPIFicoemcasa\%21.pdf/bd2e2445-10a8-46c3-a8ac-44b583906a6e SNIPI - Sistema Nacional de Intervenção Precoce na Infância.

PT.A3. DGS_008/2020, de 26/03/2020. Direção-Geral da Saúde (DGS). INFORMAÇÃO - Programa Nacional de Saúde Infantil e Juvenil e epidemia de Covid-19. Disponível em https://www.dgs.pt/normasorientacoes-e-informacoes/informacoes/informacao-n-0082020-de26032020-pdf.aspx

PT.A4. DGS 009/2020. Direção Geral da Saúde. ORIENTAÇÃO - 009/2020 de 11/03/2020. Disponível em https://www.dgs.pt/directrizes-da-dgs/ orientacoes-e-circulares-informativas/orientacao-n-0092020-de11032020-pdf.aspx

PT.A5. DGS 009-A/2020. Direção Geral da Saúde. ORIENTAÇÃO de 07/09/2020 (atualização). Disponível em https://www.dgs.pt/normasorientacoes-e-informacoes/orientacoes-e-circulares-informativas/ orientacao-n-009-a2020-de-07092020-pdf.aspx

PT.B6 CNPDPCJ\#@_2020. Comissão Nacional Promoção dos Direitos e Proteção das Crianças e Jovens. 2020. \# Proteger crianças compete a Tod@s. Disponível em: https://www.cnpdpcj.gov.pt/ documents/10182/14796/brochura+proteger+crianças+compete+a +tod\%40s/7cfd8862-864d-4a14-b1c6-5af6db4f9d79

PT.B7. IAC_SOS_2020). Instituto de Apoio à Criança. Disponível em https://iacrianca.pt/intervencao/sos-crianca

PT.A8. Decreto-Lei no 10-A/2020 de 13 de março de 2020, Diário da República, 1a série. Presidência do conselho de Ministros. Disponível em: https://dre.pt/application/conteudo/130243053

Legenda: documentos regulamentadores (letra A): lei, decreto, portaria, normas, nota técnica, recomendação governamental. Documentos orientadores (Letra B): manual, guias, orientações. 
Quadro 2. Questões analíticas e expressões-chave aplicadas aos marcos regulatórios de proteção de crianças e adolescentes vulneráveis à violência intrafamiliar. Brasil e Portugal, março a setembro de 2020.

\begin{tabular}{|c|c|}
\hline Perguntas aplicadas à leitura dos documentos & Expressões-chave \\
\hline $\begin{array}{l}\text { Quais são os órgãos e serviços de proteção referidos nos } \\
\text { documentos selecionados? }\end{array}$ & Brasil e Portugal \\
\hline $\begin{array}{l}\text { O que foi recomendado pelos órgãos e serviços de proteção } \\
\text { à criança e adolescente em risco para violência durante a } \\
\text { pandemia? }\end{array}$ & $\begin{array}{l}\text { Serviços de proteção: níveis (nacional, regional, local), } \\
\text { institucionalização, local seguro }\end{array}$ \\
\hline $\begin{array}{l}\text { Quais estratégias operacionais foram implementadas para o } \\
\text { cumprimento das recomendações? }\end{array}$ & $\begin{array}{l}\text { Estratégias: órgãos nacionais, regionais e locais, canais de } \\
\text { denúncia (telefone, aplicativos, mídias sociais), campanhas } \\
\text { nacionais, mídias sociais (acesso à informação), acolhimento, } \\
\text { profissionais de saúde e assistência social. }\end{array}$ \\
\hline \multirow{4}{*}{$\begin{array}{l}\text { Quais ações de atendimento à criança e adolescente em } \\
\text { situação de violência foram previstas pelos órgãos e serviços } \\
\text { de proteção durante a pandemia de COVID19? }\end{array}$} & $\begin{array}{l}\text { Recomendações: efetivas, de apoio, de proteção, de } \\
\text { acompanhamento. }\end{array}$ \\
\hline & $\begin{array}{l}\text { Garantia de direitos: crianças e jovens em risco, família, } \\
\text { trabalhadores }\end{array}$ \\
\hline & $\begin{array}{l}\text { Vulnerabilidade: estresse, convivência contínua, ameaça de } \\
\text { morte violência, Violência doméstica, conflito familiar/ social, } \\
\text { negligência. }\end{array}$ \\
\hline & $\begin{array}{l}\text { Pandemia de COVID-19: isolamento social, testagem, medidas } \\
\text { sanitárias. }\end{array}$ \\
\hline
\end{tabular}

serviços organizados para garantir acesso e qualidade, equipes multidisciplinares com abordagens interdisciplinares para integrar cuidados; profissionais sensibilizados para atuar em defesa dos direitos humanos, que planejam e avaliam os serviços prestados. $\mathrm{Na}$ dimensão individual, destacam-se os valores, interesses, crenças, desejos, conhecimento, atitudes, comportamento, redes sociais e de amizades, relações parentais e familiares, a saúde mental e a constituição física ${ }^{15}$.

A interpretação dos dados apontou para três núcleos de sentidos: Parcerias nacionais e locais nas ações de proteção; Apoio profissional e social na escuta atenta e proteção do direito; Modo de operacionalização das estratégias de monitoramento e acompanhamento. O último núcleo foi desdobrado em três subnúcleos: Canais de denúncia das situações de violência; Recomendações operacionais para profissionais de saúde e assistência social; Recomendações para a vigilância e segurança familiar.

\section{Aspectos éticos}

Segundo a Resolução no 510, de 7 de abril de 2016, do Conselho Nacional de Saúde, artigo 1ํ, parágrafo único, inciso III, ${ }^{16}$ os documentos regulatórios de domínio público são fontes de informações cujas pesquisas não precisam ser apreciadas pelo Sistema Conselhos de Ética em Pesquisa/ Comissão Nacional de Ética em Pesquisa (sistema CEP/ CONEP). Portanto, as fontes documentais de análise, adotadas nesta pesquisa documental, não requerem apreciação ética do projeto implementado.

\section{RESULTADOS}

Quanto ao perfil do quadro documental de análise, 17 documentos selecionados para análise, sendo nove no Brasil e oito em Portugal, estabeleciam diretrizes e ações de enfretamento da vulnerabilidade da criança à violência durante a pandemia de COVID-19. Esses documentos consistiram de leis, decreto-lei, portarias, notas técnicas, manuais contendo recomendações de governos e órgãos do controle social de proteção à infância do Brasil e de Portugal. No início da pandemia, os dois países seguiram diretrizes gerais da Organização das Nações Unidas para a Educação, a Ciência e a Cultura (UNESCO) e da Organização Mundial de Saúde (OMS), para suspender as aulas presenciais nas escolas, de modo a reduzir ou interromper o fluxo de circulação de pessoas ou mesmo impedir a aglomeração naquele ambiente, por ser uma fonte adicional de propagação do vírus.

\section{Parcerias nacionais e locais nas ações de proteção à criança e adolescente}

No Brasil, os órgãos nacionais e serviços de proteção a crianças e adolescentes em vulnerabilidade às situações de violência, durante o período da pandemia de COVID-19, envolveram dois poderes públicos.

(...) o Executivo e Judiciário. No judiciário, a segurança pública, o Sistema de Justiça e Defensoria Pública; o Ministério Público; (...) as varas da justiça da infância e da 
adolescência e o Conselho Tutelar. No executivo, o Sistema Único de Assistência Social (SUAS), representado pelos serviços da rede de proteção, sociedade civil organizada e terceiro setor; o Sistema Único de Saúde (SUS), pelos serviços públicos e privados, por meio das redes inter e intrasetoriais (saúde, assistência social e educação) (BR. A1; A2; A3, p.2/3; A6, p. 11,13/22; A7, p.12/20;B8, p.4-5,9/22)

Em Portugal, os órgãos nacionais de proteção a criança e adolescente, na instância executiva, incluíram o Sistema Nacional de Intervenção Precoce na Infância e a Comissão Nacional Proteção dos Direitos das Crianças e Jovens. (PT B2; B6, p.10) Além disso, o Instituto de Apoio à Criança, durante a pandemia, participou dos esforços dessa proteção.

[buscando] sensibilizar as Estruturas Comunitárias e a Sociedade para a Problemática da Criança/Jovem, particularmente em situação de perigo, desaparecimento, exploração ou abuso sexual. Houve ainda o envolvimento das forças de segurança e as entidades da Rede Nacional de Apoio às Vítimas de Violência Doméstica. (PT.B7; B2)

No nível local brasileiro, estabeleceram-se parcerias entre os setores da saúde e serviço social.

... profissionais da Estratégia Saúde da Família (ESF), redes de proteção e cuidado a crianças e adolescentes... que atuam nas políticas públicas, equipes técnicas $e$ representantes do Programa de Proteção a Crianças e Adolescentes Ameaçados de Morte. (BR. A1; A2, p.18/20; B8, p. 4-5,9/22; A2)

Em Portugal, nesse mesmo nível, atribuíram-se responsabilidades pela implementação das medidas de monitorização, acompanhamento e investigação de situações de violência na criança.

Núcleos de Apoio a Crianças e Jovens em Risco, aos Núcleos Hospitalares de Apoio a Crianças e Jovens em Risco, às Equipas (equipes) de Prevenção da Violência (...), associações da comunidade (associações de pais e de doentes, entre outras) e aos profissionais de saúde dos cuidados de saúde primários. (PT.A3, p.2/5)

Nos dois países, Estado, família e sociedade cumpriram com suas respectivas prerrogativas, estimulando e sensibilizando pessoas da comunidade a denunciar casos de violência. Ou seja,

qualquer pessoa (vizinho). (...) pais ou outra pessoa da familia (familiares) (BR. A2, p. 18/20; B8, p. 4-5,9/22; A2); os professores ou o orientador educacional, amigas $e$ amigos de confiança, médicos, polícias, bombeiros, CPCJ (Comissão de Proteção de Crianças e Jovens) $e$ tribunal. (BR.A2, p.18/20;B8, p. 4-5,9/22; A2; PT.B1, p.10)

\section{Apoio profissional e social na escuta atenta e proteção do direito}

Entre as estratégias recomendadas pelos órgãos e serviços de proteção à criança e adolescente no Brasil, destacam-se:

A escuta atenta e sem julgamentos... às partes, procurando agir sobre os fatores que produziram a violência, forneçam às crianças... informações sobre o que está acontecendo; a busca de formas para reduzir a vulnerabilidade social, garantindo-se o atendimento às necessidades básicas... apoio e parceria ... na aplicação da medida protetiva de acolhimento institucional para crianças e adolescentes sem retaguarda familiar. Apoio médico, psicológico, jurídico e de assistência social. (...)

Recomendaram-se que fossem investigadas as informações sobre o que está acontecendo (...), que procurasse ajuda, apoio das amigas/amigos e denunciassem para o administrador da rede social as situações de violência presencial ou virtual, respectivamente. Além disso, as recomendações foram elaboradas para que procedesse a análise e encaminhamento de denúncias de violações de direitos humanos... aos serviços dos órgãos responsáveis em funcionamento... do Executivo e Judiciário... (BR. B8, p. 9/22; (...); B9, p. 5, 13, 19, 13/22; A2, p. 2 e 3/3; B5 A1, p. 3/8; B9, p. 5, 13,19/22)

Em Portugal, as estratégias incluíram o pedido às entidades com quem a criança se relacionava no período pré-pandemia, a avaliação e intervenções, tais como:

Clubes, as salas de estudo, os amigos, os psicólogos, pedopsiquiatras, e que telefonem para as crianças e perguntem como estão, se precisam de alguma coisa, $e$ que falem mesmo com eles de forma intermitente (sem ter combinado o dia e a hora) e escutem atentamente, registem o que parece estranho, ou preocupante [...] avaliação cuidadosa, garantindo-se antecipadamente que o agressor não está a ouvir a conversa (se também estiver em casa em isolamento), e perguntar se estão a ocorrer episódios de violência... intervir para proteção imediata (...) da criança [em caso de suspeita ou ocorrência de violência]. (PT B2; B1, p.10)

\section{Modo de operacionalização das estratégias de monitoramento e acompanhamento}

No Brasil, o modo de operacionalização das estratégias recomendadas foi:

Conselhos Tutelares atendessem em regime de plantão, cabendo aos Municípios garantir recursos necessários para o trabalho remoto (internet e equipamentos), no sistema de teletrabalho temporário, teleconferências, grupos de WhatsApp (...) telefone de plantão; atendimento virtual de situações de violência. (BR. A1, p. 3/8; A2, p. 2 e 3/3) 
Quando presencial, dever-se-ia preservar as medidas sanitárias para evitar a proliferação de COVID19. (BR. A1, p. 3/8; A2, p. 2 e 3/3)

Do mesmo modo, em Portugal, recomendou-se a garantia da atividade presencial, em articulação com as áreas de Educação e Cidadania e lgualdade, respeitando-se as medidas sanitárias. (PT. B1, p.1)

\section{Canais de denúncia das situações de violência}

Tanto Brasil como Portugal apresentaram medidas concretas de proteção à criança contra a violência. As ações incluíram notificação, comunicação telefônica e uso das redes sociais, com a finalidade de notificar casos de suspeição, os quais poderiam disparar a linha de cuidado.

A notificação da suspeita de violência deveria ser feita na ficha de notificação, ... disparador da linha de cuidado (...) é obrigatória a comunicação ao Conselho Tutelar. (...) manutenção da rede de garantia de direitos... (Conselhos Tutelares, delegacias e varas especializadas). (BR. A6, p.13/22; B8, p.5/22)

Para a efetivação da notificação das situações de violência, a CNPDCR desenvolveu um modelo de ficha de comunicação de situações de perigo e que careçam de avaliação (PT. B6, p.1)

Como parte das ações dos órgãos e serviços responsáveis pela proteção da criança, explicou-se como canais de comunicação telefônica e redes sociais poderiam ser acionados para formalizar denúncias e sensibilizar a população na proteção a essas crianças.

As ligações (denúncia) podem ser feitas de todo o Brasil por...discagem gratuita, de qualquer terminal telefônico fixo ou móvel (celular). O usuário disca o número 100, passa pelo atendimento eletrônico, ... seleciona a opção desejada, é encaminhado ao atendimento humano. $O$ atendente registra a denúncia e fornece o número do protocolo. (BR. B5)

Os canais de denúncia incluíram a facultação de contactos de telefone e e-mail. Alguns destes, já existentes no período pré-pandemia em Portugal, para servir como canal de denúncia durante o período da pandemia, tais como:

SOS Criança: 116111 (...) Linha da criança: 800206656 (...) Linha Nacional de Emergência Social: 144 (disponível 24 horas por dia) ou através do 800202148 ou e-mail violência.covid@cig.gov.pt ou linha SMS 3036 (7 dias por semana, 24 horas/dia)... o número de telefone 961231111, da responsabilidade da Comissão Nacional de Promoção dos Direitos e Proteção das crianças e Jovens. (PT. B6, p.1 e 10)

Portugal recomendou à sociedade em geral o uso de redes sociais como espaço de formalização de denúncia, adotando como estratégia uma campanha nacional designada \#protegercriançascompeteatod@s, com o objetivo de sensibilizar a sociedade em geral para a necessidade de não ficar indiferente ao que se passa à sua volta. (PT. B6, p.1)

No Brasil, a sociedade foi estimulada a denunciar os possíveis abusos da rede social indicando que toda rede tem espaço de denúncia. Por lei, éobrigatória a retirada do conteúdo (...) impróprio. (BR. B4)

As recomendações operacionais para a denúncia de situações de violência contra crianças e adolescentes envolveram o registro documental, cartorial e sanitário. Nesse sentido, o Brasil buscou

disponibilizar ... promover a divulgação dos canais de denúncia nos meios de comunicação. ... que garantam interação simultânea, dispositivos eletrônicos (... celulares e computadores) com possibilidade de compartilhamento de documento, ir ao Cartório fazer uma ata notarial ou usar serviços de Prova de Autenticidade de Conteúdo Web (PACWeb), fornecidos por alguns sites, antes que a imagem saia do ar (...) após fazer o "print" de tudo... ter provas na hora da denúncia, busque a Delegacia para fazer a denúncia. (...) notificar à autoridade sanitária competente no caso de suspeita ou confirmação de violência. (BR. A2, p. 2 e 3/3; BR. A6, p. 13/22)

\section{Recomendações operacionais para profissionais de saúde e assistência social}

As recomendações para os profissionais de saúde e de assistência social para que conduzissem as entrevistas:

... fora do local de ameaça... em conformidade com o Guia de Procedimento do PPCAAM, com a presença OBRIGATÓRIA de representante da Porta de Entrada. O planejamento (do atendimento) deveria ser feito em conformidade com as diretrizes do Ministério da Saúde e que nenhum dos participantes apresentem sinais aparentes de COVID-19. (BR. A2, p.2/3)

Em Portugal, as recomendações incluíram:

... manter a articulação com diferentes programas e estruturas de suporte assim como, atualizar os contatos dos cuidadores, manter a atividade que envolve o levantamento e identificação das crianças em situação de vulnerabilidade (...) nas áreas de abrangência de cada unidade funcional do serviço de saúde. (PT. A3, p. 1/5)

No Brasil, atividades e informações sobre estratégias e práticas parentais positivas foram recomendadas para adoção pela Estratégia Saúde da Família (ESF), para:

Minimizar o surgimento de novas situações ... de crise/estresse e conflitos... manter contato direto com a criança em busca 
de sinais indicativos ...de violência, os quais devem ser informados à gerência da unidade para ... providências (...) ... manter o atendimento presencial, (...). Facilitaro contato das crianças com a rede de proteção para pedido de ajuda, zelar e proteger seus direitos (...) avaliar, monitorar (...) garantir a manutenção dos mecanismos de prevenção e repressão à violência doméstica e familiar. (BR. A1, p.3/8; A2, p. 2 e 3/3; A3, p.2/3; A6)

\section{Recomendações para a vigilância e segurança familiar}

No Brasil, a atenção especial às famílias com histórico de violência contra crianças, crianças em situação de rua, incluiu recomendações para:

(...) cuidadores/familiares usuários de álcool e outras drogas, de modo a (...) preparar e garantir seguimento ... de famílias às quais foi registrada alguma violação de direitos, ou situações graves que possam ensejar medida de acolhimento. Implementar planos de cuidados integrais em saúde física e mental a crianças (...) em situação de rua, em processo de migração, em acolhimento e/ou em privação de liberdade (...) de modo a permitir a convivência entre mãe e filhos, sua proteção e ... a privacidade desse núcleo familiar. Se ater ao cuidado e à atenção em saúde com as pessoas envolvidas na violência intrafamiliar ... verificadas outras possibilidades de proteção da criança ou adolescente sob cuidados de familiares ou pessoas próximas com vínculo de afeto e confiança, (...) condições de prover cuidado e proteção. (BR. A, p.3/8; A.7, p.12/19; B8, p.10, 13/22; A11)

Em Portugal, as recomendações consistiram em:

\begin{abstract}
... avaliar as situações garantindo a intervenção necessária e adequada... a promoção dos direitos e proteção das crianças e dos jovens, de acordo com a lei... garantida a sinalização (comunicar) das situações de perigo/risco de violência às estruturas locais da Rede Nacional de Apoio às Vítimas de Violência Doméstica... monitorização da situação de saúde destas crianças e famílias. (PT. B6, p. $1 ;$ A4, p.2/5)
\end{abstract}

Posteriormente, com a flexibilização das regras de isolamento social profilático, houve uma atualização dos procedimentos recomendados para o acolhimento de crianças e jovens nas situações de perigo e lares de proteção à infância e juventude. Assim, não se aplicam os procedimentos relativos à realização de teste laboratorial para SARS-CoV-2 e de isolamento profilático, (...) para admissão nas instituições/ Casas de Acolhimento de Crianças e Jovens em Risco. (PT.A5)
Várias medidas de proteção social na doença e parentalidade foram previstas, tanto no Brasil quanto em Portugal, tais como subsídios, afastamento do trabalho e segurança social no trabalho.

Subsídios de assistência a filho e a neto ... menor de 12 anos ou, independentemente da idade, com deficiência ou doença crónica; a atribuição do subsídio para assistência (...), não depende de prazo de garantia ${ }^{\dagger}$. (PT. A7)

O trabalhador tem direito a receber um apoio excecional mensal, ou proporcional, ... pago em (duas) partes iguais pela entidade empregadora e pela segurança social... percebidos uma vez e não simultaneamente por ambos os progenitores, independentemente do número de filhos ou dependentes a cargo. Falta justificada do trabalhador sem perda de direitos... do acompanhamento de isolamento profilático (protegido pelo) regime geral de segurança social, motivado por situações de grave risco a saúde pública (...); decorrentes de suspensão das atividades letivas e não letivas presenciais em estabelecimento escolar ou equipamento social de apoio à primeira infância ou deficiência (...). (PT, A8)

Implementar políticas de garantia de afastamento do trabalho de pais e mães de crianças em idade escolar que estejam atuando em serviços essenciais (...) emprego e renda, auxílios emergenciais e segurança alimentar para famílias em situação de risco e vulnerabilidade social. (BR. B8, p.10, 13/22)

\section{DISCUSSÃO}

Quanto às parcerias nacionais e locais nas ações de proteção a criança e adolescente, destaca-se o apoio interinstitucional para o desenvolvimento de ações articuladas na proteção ao direito à vida e à dignidade. Especialmente para a proteção de crianças sem retaguarda familiar, recomendou-se o envolvimento de diferentes setores governamentais na garantia da aplicação da medida protetiva de acolhimento institucional.

A violência na infância em momentos de crise sanitária apresenta um caráter multisistêmico e exige intervenção intersetorial para mitigar os fatores geradores das vulnerabilidades individuais e coletivas (programáticas e sociais) ${ }^{14,15}$.

No Brasil, para garantir as ações de proteção a crianças e adolescentes ameaçados de morte, estabeleceram-se diretrizes para a condução de entrevistas de avaliações em local seguro, fora de ameaça e na presença de representante da Porta de Entrada. Os critérios de avaliação para o ingresso no Programa de Proteção à Criança e Adolescente Ameaçado de Morte variou em função da logística local, incluindo acesso ao local

\footnotetext{
† O prazo de garantia é uma das condições chave de acesso ao subsídio de desemprego. É uma medida temporal que permite saber se uma pessoa tem direito ao subsídio enquanto está desempregado. Fonte: subsídio de desemprego (Atualizado em 15/02/2021). Segurança Social 2021, 15p Disponível em: http://www.seg-social.pt/subsidio-social-de-desemprego
} 
seguro por deslocamento terrestre ou aéreo. Recomendaram-se, também, a hospedagem provisória, a locação de imóvel, a vaga em acolhimento institucional para casos de proteção sem acompanhamento de responsável legal, apoio e acompanhamento do sistema de garantia de direitos de proteção. Além disso, buscou-se assegurar a continuidade das ações de proteção às crianças que vivem sob ameaça de morte, provendo acesso seguro e que os profissionais que não sejam do grupo de risco para COVID-19 ou estejam cuidando de pessoas deste grupo.

As Comissões de Proteção de Crianças e Jovens são estruturas reguladas pela legislação portuguesa como forma de resposta social no enfrentamento das vulnerabilidades programáticas. Nesse sentido, há uma rede de serviços da comunidade que estabelece parceria com o Estado. A legislação portuguesa reconhece a vulnerabilidade individual e o risco elevado de dano e de impacto negativo da violência na vida das crianças, paradoxalmente perpetuada pelos adultos ou responsáveis legais, cuidadores e que, no lugar de cuidar e proteger, funcionam como modelos de comportamentos negativos, suscetíveis de perpetuar a violência no presente e no futuro. Essas Comissões orientam seu processo de trabalho, adotando-se o modelo de promoção e proteção aplicado por instituições não judiciárias ${ }^{17}$.

Nesse contexto, a situação da criança e sua família merece atenção, especialmente no que concerne às adaptações impostas neste período que proporcionaram significativas mudanças na dinâmica relacional, o surgimento de novos delineamentos das rotinas familiares, reforçando e/ou agravando situações de violência já vivenciadas e trazendo outras possibilidades de exposição à violência ${ }^{18,19}$.

A recomendação para assegurar o apoio profissional e social com escuta atenta e protetiva do direito da criança e adolescente foi um achado relevante na análise dos dispositivos regulatórios expedidos pelos dois países. Entretanto, o não funcionamento de creches e jardins de infância em Portugal e a educação infantil no Brasil representaram estruturas organizadas de proteção às vulnerabilidades individuais de crianças pequenas indisponíveis durante a pandemia. Nesse sentido, a intervenção de profissionais com domínio na abordagem da violência contra a criança também foi interrompido com o isolamento social. Esse advento dificultou a identificação mais prontamente dos casos de violência e aquelas crianças mais afetadas pela violência deixou de se beneficiar do apoio de especialistas em aconselhamento familiar ou de programas para o tratamento de traumas infantis, a expressar emoções e lidar com o estresse pós-traumático.

A intervenção das comissões de proteção de crianças e jovens assumiu o lugar do atendimento imediato individual, porque não foi possível às instituições atuarem de forma adequada e suficiente para afastar crianças e jovens das fontes de ameaças e perigo que poderiam estar expostos. Quando criança, adolescente e jovem estão em situação de vulnerabilidade, especialmente quando pais, representante legal ou guardião são os potenciais agressores, faz-se necessário intervenção institucional do Estado por meio dos órgãos nacionais e locais e dos profissionais (educação, saúde e assistência social), para protegê-las ${ }^{19}$.
Os diferentes dispositivos normativos recomendaram escuta atenta, afastamento das fontes de ameaças e perigos, acolhimento institucional, intervenção de promoção e proteção aos direitos da criança e de famílias socialmente vulnerabilizadas, além do acompanhamento, vigilância e denúncia. Da mesma forma, os documentos destacaram a manutenção do acompanhamento $e$ monitoramento presenciais pelas equipes de saúde e assistência social de ambos os países, visando ao seguimento pelos serviços de saúde e vigilância às redes sociais. Disponibilizaram-se canais de comunicação com as autoridades competentes para denúncia e notificação de casos de violência.

Sobre o modo de operacionalização das estratégias de monitoramento e acompanhamento, ambos os países previram o acompanhamento presencial às crianças e adolescentes em situação de violência. No que concerne ao monitoramento e acompanhamento das crianças expostas às situações de violência em diferentes contextos de vulnerabilidade social, recomendou-se atenção especial às famílias e crianças com maior exposição a vulnerabilidades e risco de agravos. Ou seja, aquelas com histórico de violência, crianças em situação de rua e em casas com cuidadores usuários de álcool e outras drogas, além das que vivem nas fronteiras, as migrantes e em contextos de aglomeração e afastamento familiar.

As crianças brasileiras mais vulneráveis à violência são: aquelas expostas a conflitos domésticos decorrentes do confinamento prolongado, redução de renda e escassez de recursos; as que vivem em situação de rua; as migrantes; as dos povos do campo, floresta e águas. Quanto às iniciativas brasileiras visando à proteção desse grupo infantil, destacam-se o acompanhamento e o monitoramento pelos órgãos responsáveis pela sua proteção.

Em Portugal, as respostas sociais de acolhimento a crianças e jovens expostos à violência integram instituições de apoio, como os centros (alguns especializados) e núcleos de atendimento, linhas de emergência, entre outros. Destacam-se a divulgação de materiais informativos e manuais com orientações de intervenção para fomentar a preparação de profissionais no atendimento desse grupo populacional exposto à violência ou outras situações de risco ${ }^{17}$.

A despeito da diferença de extensão territorial e demográfica dos dois países, tem-se em comum a matriz cultural latina, que nos aproxima da ideologia da "autoridade parental" na educação de crianças, em decorrência das relações paterno-filiais.

No Brasil, a Lei da Palmada, que alterou o Estatuto da Criança e do Adolescente ${ }^{10}$, que delimita os castigos e a disciplina no exercício da autoridade parental, entendendo-a como obrigação fundamental de prover o sustento e a formação geral segundo as necessidades dos filhos e não os arbítrios do provedor (pai, mãe ou pais) ${ }^{20}$. Práticas parentais de educação da criança baseadas em atos violentos geram uma cultura de silêncio intrafamiliar. Por razões culturais, o país ainda enfrenta uma baixa aplicabilidade da Lei, o que contribui para explicar a baixa notificação quando a criança circula menos nos serviços educacionais e de saúde ${ }^{20}$. 
Do mesmo modo que no Brasil, a violência contra a criança e adolescente em Portugal está associada ao agressor parental ou de entes significativos (pais ou cuidadores). O lugar onde a criança se expõe mais à violência contribui para compreender as relações de poder que são complexas e refletem as diversas formas de opressão, desigualdades e discriminação da infância ${ }^{21}$.

Parece haver uma naturalização da violência como recurso pedagógico e intergeracional. Uma maior convivência no ambiente doméstico, muitas vezes, revela o fenômeno da violência intrafamiliar, na qual o agressor reproduz ações violentas anteriores e culturalmente aprendidas como uma medida disciplinar e coercitiva, estabelecendo uma relação desigual de poder que transpõe sucessivas gerações. Por ser um fenômeno multifacetado, está relacionado a fatores sociais, culturais e econômicos que afeta as relações interpessoais ${ }^{21,22}$.

Conselhos Tutelares, serviços de saúde e outros serviços da rede de proteção social implementaram ações de prevenção, acompanhamento e monitoramento de fontes de conflitos que pudessem desencadear violência contra a criança. Há um alerta, ainda, para a necessidade de se garantir a proteção integral às crianças e o tratamento para a COVID-19. As ações das equipes da ESF, atividades e informações sobre estratégias e práticas parentais positivas foram recomendadas para diminuir fontes de conflito e as situações de violência no ambiente doméstico, especialmente quando houvesse impossibilidade do isolamento social completo em instituições de acolhimento, crianças em situação de rua ou de violência doméstica.

A recomendação para uso de novas tecnologias foi estratégica para assegurar o telemonitoramento, sendo que, em situações que houvesse necessidade de intervir para proteção imediata da criança, poderia ser necessário retirá-la do domicílio e transferi-la para uma instituição de acolhimento.

Estudo desenvolvido no estado de Santa Catarina verificou diminuição considerável $(55,3 \%)$ nos registros das notificações de violência à criança e ao adolescente durante o período da pandemia de COVID-19. Isso pode estar atrelado a algum tipo de dificuldade de se fazer a denúncia ou ao acesso às redes de cuidado, proteção e assistência. Isso pode estar atrelado às dificuldades de acesso aos canais de denúncia ou às redes de cuidado, proteção, assistência à saúde e social. Todavia, o aumento da violência doméstica, bem como a redução das denúncias, pode se configurar em uma situação irreparável que se revela para além da ameaça imediata da COVID-19, com consequências assoladoras no contexto da saúde pública global ${ }^{23-25}$.

O confinamento foi potencializador de vulnerabilidades que aumentaram as necessidades de saúde e a exposição à violência intrafamiliar e social. Não obstante, mesmo que a estratégia do isolamento social tenha sido implementada como medida eficaz para o controle da disseminação da COVID-19, as repercussões sociais, econômicas e psicológicas afetam a vida das pessoas, podendo corroborar para situações de violência.

Apesar dos esforços investidos pelos dois países, houve maior predisposição à redução da notificação de casos de violência aos órgãos responsáveis. Consequentemente, houve um maior risco de escassez de dados, pois reduziram-se os espaços de convivência facilitadores da identificação e denúncias, como creches e escolas, que permaneceram fechadas no período da pandemia. A invisibilidade da violência se apresenta como um efeito adverso ao isolamento social provocado pela pandemia. Portanto, o agravamento dessa situação complexa e multidimensional carrega consigo variadas fontes de tensão e conflito cotidiano, as quais exigiram recomendações operacionais para profissionais de saúde e assistência social.

OUNICEF alerta que crianças em isolamento social apresentam maior vulnerabilidade à violência intrafamiliar como resultado dos fatores que afetam a dinâmica relacional da família. Pode haver aumento do estresse dos pais ou responsáveis, ao buscar alternativas para a manutenção do trabalho remoto e do cuidado às crianças diante da escassez ou interrupção do acesso às redes de apoio familiares e comunitárias, além da perda ou redução da renda familiar ${ }^{19}$. Dificuldades econômicas e escassez de recursos essenciais à sobrevivência se somam ao distanciamento social e confinamento como fontes geradoras de estresse familiar ${ }^{19}$.

A situação de violência à criança pode se acentuar com o afastamento dos colegas e professores da escola, um espaço de relações interpessoais com disponibilidade de serviços mais seguros. Desse modo, crianças refugiadas, migrantes, que vivem em situação de rua, ambientes de favelas e áreas de conflito e com deficiência, são mais vulneráveis ${ }^{26}$. Além disso, o aumento da vulnerabilidade econômica das famílias pode acarretar o aumento do trabalho infantil, entre outros ${ }^{27}$.

Independentemente de os países serem mais economicamente desenvolvidos ou não, crianças que vivem em contexto de pobreza, situação de rua, refugiadas, migrantes, ambientes de favelas, áreas de conflito com risco de morte, deficiência ou outras necessidades sociais e de saúde específicas são mais vulneráveis à violência. Além disso, o aumento da vulnerabilidade econômica das famílias pode acarretar o aumento do trabalho infantil, entre outros ${ }^{26-28}$.

Tanto no Brasil como em Portugal há a preocupação com a notificação e investigação das situações de vulnerabilidade da criança e adolescente à violência, particularmente aquelas em situação de perigo e risco de morte. As medidas envolvem a escuta atenta, sem julgamento e o registro da mudança comportamento da criança, devendo ser realizada por todos os seus contatos relacionados à saúde, às atividades escolares e lúdicas.

Entre as medidas de proteção social na doença da COVID-19, foram estabelecidas proteção à parentalidade, que incluíram subsídios de assistência a filho e/ou netos em Portugal, e o auxílio emergencial no valor de $\mathrm{R} \$ 600,00$ para as famílias brasileiras com crianças e adolescentes cujos pais ficaram sem fonte de renda, não descontando os dias de trabalho ausentes, entre outras medidas.

Em função das respostas políticas, os riscos de perda repentina de renda ou acesso à proteção social há consequências que são difíceis de estimar e constituem um desafio na identificação de todos aqueles que podem se tornar vulneráveis, entre eles as crianças. ${ }^{29} \mathrm{~A}$ vulnerabilidade em saúde reconhece as conjunturas macrossociais associadas à dimensão pessoal necessária ao enfrentamento das situações vulnerabilizantes. Tal olhar não 
se distancia de uma acepção mais abrangente em relação a contextos desiguais e socialmente injustos, porquanto refina a importância da capacidade de elaboração e interesse dos sujeitos para a incorporação dessa temática em seu dia a dia ${ }^{19}$

Várias ferramentas foram disponibilizadas em ambiente remoto para as denúncias de violência contra a criança e o monitoramento de casos, em ambos os países (telefone, redes sociais, campanhas de sensibilização, etc). Canais de comunicação para denúncia de violência (Disque 100, aplicativos, e-mail e Ouvidoria) foram estabelecidos no plano da proteção à violência contra a criança.

Há limitações dos próprios serviços de proteção, dificuldades de deslocamento e de acesso da população à tecnologia de comunicação que podem influir sobre o uso dos canais disponibilizados pelas estruturas governamentais nesses tempos de pandemia. Para além da disponibilização das ferramentas supramencionadas, é imprescindível a sua divulgação e sensibilização da sociedade sobre os diversos canais de denúncia e notificação, com a finalidade de proteger as vítimas e romper o ciclo da violência ${ }^{23}$.

As consequências da violência podem se perdurar ao longo da vida, afetando o desenvolvimento potencial do indivíduo com danos irreversíveis e trazendo impactos que se estendem às famílias e à comunidade $^{1,28,29}$. A violência se apresenta como uma realidade de risco elevado de ocorrência, principalmente na fase aguda da crise, o que requer medidas imediatas para o rompimento deste ciclo.

\section{CONSIDERAÇÕES FINAIS E IMPLICAÇÕES PARA A PRÁTICA DE ENFERMAGEM}

A pandemia de COVID-19 evidenciou a exposição de crianças e adolescentes a situações de violência intrafamiliar e maior risco de subnotificação de casos. As medidas de distanciamento social e o confinamento domiciliar propiciaram maior convivência em família, que, combinada a fatores geradores de conflito, aumentaram a vulnerabilidade individual das crianças, adolescentes e jovens. Entre eles, destacam-se os relacionados à criança, ao estresse, à restrição da mobilidade e acesso a equipamentos sociais, e à interrupção da rotina na escola. Os relacionados à família, que contribuíram para aumentar a vulnerabilidade social, foram o trabalho remoto, a sobrecarga nas tarefas domésticas e a escassez de renda ou desemprego. Para o enfrentamento dessas vulnerabilidades individuais e sociais, os órgãos governamentais nacionais e internacionais disponibilizaram documentos contendo recomendações, estratégias e garantias de proteção a este grupo social, de modo a minimizar as vulnerabilidades programáticas.

Tais documentos, expedidos pelos governos de Brasil e Portugal, determinaram ações dirigidas às instituições, profissionais de saúde, familiares e sociedade civil com vistas à continuidade da proteção, da prevenção, em uma perspectiva multiprofissional, intra e intersetorial. Recomendaram-se, também, estratégias para a interrupção do ciclo da violência às crianças e famílias em situação de maior vulnerabilidade, especialmente aquelas com história pregressa de práticas violentas, uso de drogas e álcool, bem como crianças em situação de rua, migração, as que vivem em locais de maior aglomeração populacional e as ameaçadas de morte.

Consequentemente, como implicação do estudo, é preciso fortalecer ações integradas, maior empenho e resolutividade dos órgãos competentes para intervir, precocemente, na identificação de violações aos direitos humanos e efetividade da garantia à integralidade da proteção de crianças e adolescentes. Nesse sentido, vizinhos, amigos, professores, educadores e demais profissionais em contato próximo com a criança precisam se manter alertas para os diversos sinais e denunciar situações de violência pelos canais disponíveis. São iniciativas que podem interromper ciclos de violência, muitas vezes culturalmente perpetuados. Com a vulnerabilidade da população infantil no cenário de pandemia de COVID-19, é necessário que o Estado proteja crianças, adolescentes e jovens, assegurando a oferta de serviços organizados para proporcionar assistência integral à saúde da criança, em seus aspectos físico, psíquico e social.

Entre as limitações do estudo, destaca-se a metodológica relacionada a pesquisas com documentos, sem escuta de profissionais que atuam na proteção de crianças e adolescentes vulneráveis à violência durante a pandemia. A limitação geográfica se refere à base documental de dois países de língua portuguesa, com sistemas universais de proteção social e saúde cujos resultados podem não se aplicar a outros países.

\section{FINANCIAMENTO}

Conselho Nacional de Desenvolvimento Científico e Tecnológico (CNPQ), concessão a Ivone Evangelista Cabral de bolsa de produtividade, Processo no 303149/2019-1, e financiamento do projeto de pesquisa do Edital Universal Chamada MCTIC/CNPq № 28/2018 - Processo no 430213/2018-2; Bolsa do Programa Institucional de Bolsa de Iniciação Científica da UFRJ, concedida a Yan do Rosario Nunes, ano 2020-2021.

\section{CONTRIBUIÇÕES DOS AUTORES}

Desenho do estudo. Ivone Evangelista Cabral. Lia Leão Ciuffo. Márcia Pestana Santos. Maria de Lurdes Lomba.

Coleta ou produção dos dados. Ivone Evangelista Cabral. Lia Leão Ciuffo. Márcia Pestana Santos. Maria de Lurdes Lomba.

Análise de dados. Ivone Evangelista Cabral. Lia Leão Ciuffo.

Márcia Pestana Santos. Yan do Rosario Nunes. Maria de Lurdes Lomba.

Interpretação de dados. Ivone Evangelista Cabral. Lia Leão Ciuffo. Márcia Pestana Santos. Yan do Rosario Nunes. Maria de Lurdes Lomba.

Redação e revisão crítica do manuscrito. Ivone Evangelista Cabral. Lia Leão Ciuffo. Márcia Pestana Santos. Yan Nunes Rosario. Maria de Lurdes Lomba.

Aprovação da versão final do conteúdo a ser publicado. Ivone Evangelista Cabral. Lia Leão Ciuffo. Márcia Pestana Santos. Yan do Rosario Nunes. Maria de Lurdes Lomba. 
Concordância em responsabilizar-se por todos os aspectos do trabalho assegurando que questões relacionadas a acurácia ou integridade de quaisquer partes do trabalho serão adequadamente investigadas e resolvidas. Ivone Evangelista Cabral. Lia Leão Ciuffo. Márcia Pestana Santos. Yan do Rosario Nunes. Maria de Lurdes Lomba.

\section{EDITOR ASSOCIADO}

Antonio José Almeida Filho (1)

\section{EDITOR CIENTÍFICO}

Marcelle Miranda da Silva (1)

\section{REFERÊNCIAS}

1. World Health Organization. Global status report on preventing violence against children [Internet]. Geneva: WHO; 2020 [citado 2020 jul 23]. Disponível em: https://www.who.int/publications/i/ item/9789240004191

2. United Nations Children's Fund. Protecting children from the violence during the time of COVID-19: disruptions in prevention and response services [Internet]. New York: UNICEF; 2020. 10 p. [citado 2020 jul 23] Disponível em: https://www.unicef.org/media/74146/file/Protectingchildren-from-violence-in-the-time-of-covid-19.pdf\%203

3. United Nations Educational. Educação: da interrupção à recuperação [Internet]. Paris: UNESCO; 2020 [citado 2020 jul 23]. Disponível em: https://pt.unesco.org/covid19/educationresponse

4. Campbell S, Carnevale F. Injustices confronted by children \& youth during the COVID-19 pandemic [Internet]. Medium; 2020 [citado 2020 jul 23]. Disponível em: https://medium.com/@sydney.campbell/ injustices-confronted-by-children-youth-during-the-covid-19-pandemica0d725ddd50e

5. Ministério da Mulher, da Família e dos Direitos Humanos (BR). Covid-19: Cai número de denúncias de violações contra crianças e adolescentes no Disque 100 [Internet]. Brasília: Ministério da Mulher, da Família e dos Direitos Humanos; 2020 [citado 2020 jul 23]. Disponível em: https://www. gov.br/mdh/pt-br/assuntos/noticias/2020-2/julho/covid-19-cai-numero-dedenuncias-de-violacoes-contra-criancas-e-adolescentes-no-disque-100

6. Ouvidoria Nacional de Direitos Humanos. Disque Direitos Humanos. Relatório 2019 [Internet]. Brasília: Ouvidoria Nacional de Direitos Humanos; 2020 [citado 2020 jul 23]. p. 42-61. Disponível em: https:// www.gov.br/mdh/pt-br/assuntos/noticias/2020-2/junho/balancoanual-disque-100-atendeu-2-7-milhoes-de-ligacoes-em-2019/ copy_of_Relatorio_Disque_100_final.pdf

7. Associação Portuguesa de Apoio à Vítima. Estatísticas APAV. Relatório Anual 2019 [Internet]. Lisboa: Associação Portuguesa de Apoio à Vítima; 2020 [cited 2020 dez 17]. 18 p. Disponível em: https://apav.pt/apav_v3/ images/pdf/Estatisticas_APAV-Relatorio_Anual_2019.pdf

8. Portugal. Instituto da Segurança Social. Relatório CASA 2019 [Internet]. [citado 2020 dez 17]. Disponível em: http://www.seg-social.pt/ documents/10152/17405298/Relatório\%20CASA\%202019/0bf7ca2bd8a9-44d2-bff7-df1f111dc7ee

9. OPAS/OMS Brasil. Países estão falhando em prevenir violência contra crianças, alertam agências [Internet]. 2020 [citado 2020 jul 23]. 5 p. Disponível em: https://www.paho.org/bra/index.php?option=com_co ntent\&view=article\&id=6201: paises-estao-falhando-em-prevenirviolencia-contra-criancas-alertam-agencias $\&$ ltemid $=820$

10. Senado Federal (BR). Estatuto da criança e do adolescente. Brasília: Senado Federal, Coordenação de Edições Técnicas; 2017. 115 p.

11. Ferreira JM. Sistema de protecção à infância em Portugal - Uma área de intervenção e estudo do Serviço Social. Rev. Katál. [Internet].2010 jul./dez; [citado 2020 jul 23];13(2):229-39. Disponível em: https://periodicos.ufsc. br/index.php/katalysis/article/view/S1414-49802010000200010/15106
12. Sá-Silva JR, de Almeida CD, Guindani JF. Pesquisa documental pistas teóricas e metodológicas. Rev Bras História Ciências Sociais. 2009;1(1):1-15.

13. Fonseca MJM. Introdução à hermenêutica de Paul Ricoeur. Millenium Journal of Education, Technologies, and Health [Internet]. 2009; [citado 2020 jul 23];36(14):1-27. Disponível em: https://revistas.rcaap.pt/ millenium/article/view/8284

14. Malagón-Oviedo RA, Czeresnia D. O conceito de vulnerabilidade e seu caráter biossocial. Interface (Botucatu). 2015;19(53):237-50. http:// dx.doi.org/10.1590/1807-57622014.0436.

15. Ayres JR, Paiva V, França Jr I, Gravato N, Lacerda R, Della Negra M et al. Vulnerability, Human Rights, and Comprehensive Health Care Needs of Young People Living With HIV/AIDS. Am J Public Health. 2006;96(6):10016. http://dx.doi.org/10.2105/AJPH.2004.060905. PMid:16449593.

16. Guerriero ICZ, Minayo MC. A aprovação da Resolução CNS nº 510/2016 é um avanço para a ciência brasileira. Saude Soc. 2019 dez;28(4):299310. http://dx.doi.org/10.1590/s0104-12902019190232.

17. Tomás CA, Fernandes N, Sani Al, Martins PCA. (in) visibilidade das crianças na violência doméstica em Portugal. SER Social. 2018;20(43):387410. http://dx.doi.org/10.26512/ser_social.v20i43.18867.

18. Usher K, Bhullar N, Durkin J, Gyamfi N, Jackson D. Family violence and COVID-19: Increased vulnerability and reduced options for support. Int J Ment Health Nurs. 2020;29(4):549-52. http://dx.doi.org/10.1111/ inm.12735. PMid:32314526.

19. The Alliance for Child Protection in Humanitarian Action. Nota Técnica: Proteção da Criança durante a Pandemia do Coronavírus, Versão 1 [Internet]. Brasília: UNICEF; 2020 [citado 2020 jul 23]. Disponível em: https://www.unicef.org/brazil/media/7516/file/nota-tecnica_protecaoda-crianca-durante-a-pandemia-do-coronavirus.pdf

20. Souza IA, Belini LF. Autoridade parental e lei da palmada. RBDCivi [Internet]. 2018; [citado 2021 mar 5];17: 65-81. Disponível em: https:// rbdcivil.ibdcivil.org.br/rbdc/article/view/271/229

21. World Health Organization. World report on violence and health [Internet] Geneva:WHO;2002 [citado 2020 jul 23]. Disponível em: https://www.who. int/violence_injury_prevention/violence/world_report/en/summary_en.pdf

22. Hino P, Takahashi RF, Nichiata LYI, Apostólico MR, Taminato M, Fernandes $\mathrm{H}$. Interfaces of vulnerability dimensions in violence against children. Rev Bras Enferm. 2019;72(Suppl 3):343-7. http://dx.doi. org/10.1590/0034-7167-2018-0463.

23. Platt VB, Guedert JM, Coelho EBS. Violence against children and adolescents: notification and alert in times of pandemic. Rev Paul Pediatr. 2021;39:e2020267. http://dx.doi.org/10.1590/1984-0462/2021/39/2020267. PMid:33146295.

24. Thomas EY, Anurudran A, Robb K, Burke TF. Spotlight on child abuse and neglect response in the time of COVID-19. Lancet Public Health 2020;5(7):e371. http://dx.doi.org/10.1016/S2468-2667(20)30143-2. PMid:32619538.

25. Chandan JS, Taylor J, Bradbury-Jones C, Nirantharakumar K, Kane E, Bandyopadhyay S. COVID-19: a public health approach to manage domestic violence is needed. Lancet Public Health. 2020;5(6):e309. http://dx.doi.org/10.1016/S2468-2667(20)30112-2. PMid:32401709.

26. World Health Organization. Joint Leaders' statement - violence against children: a hidden crisis of the COVID-19 pandemic [Internet]. Geneva: WHO; 2020 [citado 2020 jul 23]. Disponível em: https://www.who.int/ news-room/detail/08-04-2020-joint-leader-s-statement---violenceagainst-children-a-hidden-crisis-of-the-covid-19-pandemic

27. Carmo ME, Guizardi FL. The concept of vulnerability and its meanings for public policies in health and social welfare. Cad. Saúde Pública. 2018;34(3):e00101417. https://doi.org/10.1590/0102-311x00101417.

28. The Lancet. Redefining vulnerability in the era of COVID-19 [editorial] The Lancet. 2020;395(10230):1089. https://doi.org/10.1016/S01406736(20)30757-1.

29. Pereda N, Díaz-Faes DA. Family violence against children in the wake of COVID-19 pandemic: a review of current perspectives and risk factors. Child Adolesc Psychiatry Ment Health. 2020;14:40. http://dx.doi. org/10.1186/s13034-020-00347-1. PMid:33088340. 